\title{
Effect of Growth Conditions on Phenazine Production by Pseudomonas phenazinium
}

\author{
By ANN J. M. MESSENGER*† AND JOHN M. TURNER \\ Department of Biochemistry, University of Liverpool, P.O. Box 147, Liverpool L69 3BX, U.K.
}

(Received 4 October 1982)

Phenazine production by Pseudomonas phenazinium was not reduced by high concentrations of phosphate during batch culture (cf. Pseudomonas aeruginosa) but was inhibited by high concentrations of $\left(\mathrm{NH}_{4}\right)_{2} \mathrm{SO}_{4}$ (1 to $\left.10 \mathrm{~g} \mathrm{l}^{-1}\right)$. In carbon-, sulphate-, magnesium- and potassiumdepleted medium phenazine production paralleled growth. In continuous culture, phenazine production occurred over a wide range of growth rates under various nutrient-limiting conditions. At low growth rates cell resources were conserved and cell mass was maintained at the expense of secondary metabolite production. Low oxygen tensions and the addition of phenylalanine $\left(0 \cdot 1 \mathrm{~g}^{-1}\right)$ each inhibited phenazine production.

\section{INTRODUCTION}

Ten different phenazines are produced by Pseudomonas phenazinium wild-type. The major one is the purple pigment, iodinin, whereas 9 other phenazines are only produced in trace amounts. Evidence has been obtained for a branched biosynthetic pathway (Byng \& Turner, 1976, 1977) (Fig. 1). Mutants with altered patterns of phenazine production are available (Byng $\&$ Turner, 1976). Phenazines are secondary products of the shikimate pathway, chorismate being the branchpoint compound (Calhoun et al., 1972; Longley et al., 1972).

Although phenazines are produced in copious quantities by $P$. phenazinium under certain conditions, e.g. growth on a minimal salts medium containing L-threonine (Bell \& Turner, 1973; Byng \& Turner, 1975), their significance is uncertain.

Several reports have described the effects of growth conditions on phenazine production by pseudomonads, e.g. medium composition in the case of Pseudomonas aureofaciens (Korth, 1973, 1974) and temperature and oxygen saturation in the case of Pseudomonas aeruginosa (Kanner et al., 1978). Low concentrations of phosphate in the growth medium for $P$. aeruginosa stimulated pyocyanin production (Ingledew \& Campbell, 1969). The advantages to the producing organism of phenazine production have never been assessed.

In this paper we describe the effects of growth conditions on phenazine production by $P$. phenazinium in batch and continuous culture.

\section{METHODS}

Micro-organisms. Pseudomonas phenazinium (NCIB 11027) was obtained from the National Collection of Industrial and Marine Bacteria, Torry Research Station, Aberdeen, U.K. It was originally isolated in this laboratory from a soil sample (Bell \& Turner, 1973). Several mutants with altered patterns of phenazine production have been isolated (Byng \& Turner, 1976). Mutant D34 was chosen for growth in continuous culture as it produced soluble phenazines from both branches of the phenazine pathway (Fig. 1). The major phenazine produced by the wild-type pseudomonad is water-insoluble iodinin which aggregates, adheres to surfaces and precludes homogeneous sampling during growth.

$\dagger$ Present address: Department of Biochemistry, University of Hull, Hull HU6 7RX, U.K. 
Shikimate $\rightarrow$ Chorismate $\rightarrow$ Aromatic amino acids

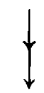

Phenazine 1,6-dicarboxylate

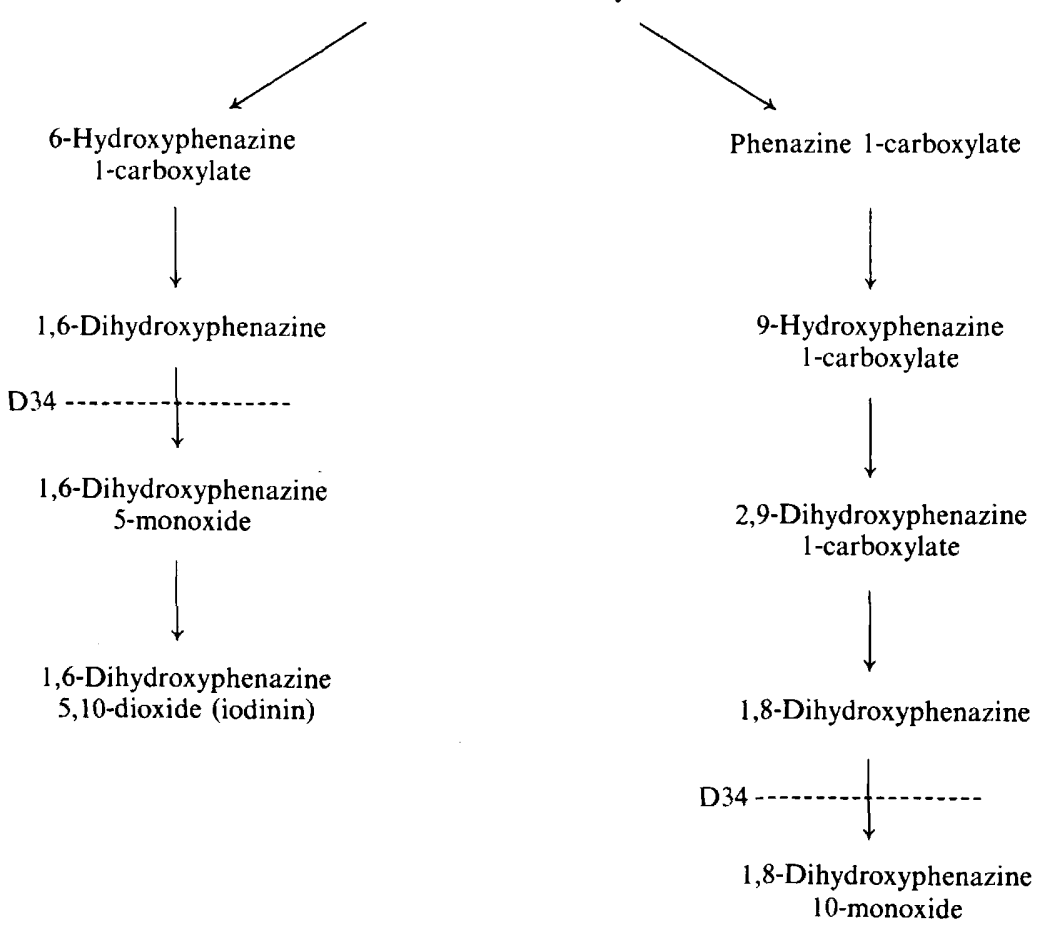

Fig. 1. Scheme for phenazine biosynthesis in P. phenazinium (from Byng \& Turner, 1977). The probable positions of lesions in mutant D34 are indicated. This strain produces no phenazine $N$-oxides and it is possible that the same enzyme is responsible for oxidation of the 1,6- and 1,8-dihydroxyphenazines (Byng \& Turner, 1976). Thus both lesions in the pathway may be produced by a single mutation. However, further evidence is needed to clarify this.

Media and culture conditions. For phenazine production $P$. phenazinium strains were grown in liquid culture on basal carbon-limiting medium containing $2 \mathrm{~g} \mathrm{~L}$-threonine, $1.2 \mathrm{~g} \mathrm{Na}_{2} \mathrm{SO}_{4}, 0 \cdot 1 \mathrm{~g} \mathrm{MgSO}_{4} .7 \mathrm{H}_{2} \mathrm{O}$ and $8 \cdot 5 \mathrm{~g} \mathrm{KH}_{2} \mathrm{PO}_{4}$ made up to 1 litre with glass-distilled water and adjusted to $\mathrm{pH} 5.5$ with concentrated $\mathrm{NaOH}$. All other media were modifications of this. Phosphate-depleted medium contained $7.5 \mathrm{~g}$ morpholinoethane sulphonate buffer and $0.25 \mathrm{~g} \mathrm{KH}_{2} \mathrm{PO}_{4} \mathrm{1}^{-1}$; nitrogen-depleted medium contained $2.5 \mathrm{~g}$ glycerol and $0.5 \mathrm{~g}$ threonine $\mathrm{l}^{-1}$; sulphate-depleted medium contained $0 \cdot 1 \mathrm{~g} \mathrm{MgCl}^{-1}$ to replace $\mathrm{MgSO}_{4}$; magnesium-depleted medium contained $0 \cdot 1 \mathrm{~g} \mathrm{Na}_{2} \mathrm{SO}_{4} \mathrm{l}^{-1}$ to replace $\mathrm{MgSO}_{4}$ and potassium-depleted medium contained $8.5 \mathrm{~g} \mathrm{NaH}_{2} \mathrm{PO}_{4} \mathrm{l}^{-1}$ to replace $\mathrm{KH}_{2} \mathrm{PO}_{4}$.

Liquid cultures $(100 \mathrm{ml})$ were grown in $250 \mathrm{ml}$ conical flasks and shaken at $30^{\circ} \mathrm{C}$ on a gyratory shaker. Growth of cultures was measured spectrophotometrically at $600 \mathrm{~nm}$. Pseudomonas phenazinium mutant D34 was grown in continuous culture in a 2.51 chemostat. The steady state was usually reached when 4 to 5 culture volumes of medium had passed through the vessel and was monitored by measuring the absorbance of culture samples. At each steady state a $1 \mathrm{ml}$ sample was taken for viable cell counts, a $20 \mathrm{ml}$ sample for phenazine estimation and a $50 \mathrm{ml}$ sample for cell mass estimation. Chemostat temperature was maintained at $30^{\circ} \mathrm{C}$ by a temperature probe and $\mathrm{pH}$ was not controlled during growth. Oxygen saturation was measured by an oxygen probe. Complete saturation was achieved by bubbling compressed air through the sterile medium at a rate of $4.51 \mathrm{~min}^{-1}$ until the oxygen level remained constant. Oxygen saturation is expressed as a percentage of this value.

Measurement of cell dry mass. A modification of the method of Hadijpetrou et al. (1964) was used. Samples $(50 \mathrm{ml})$ were centrifuged at $12000 \mathrm{~g}$ for $20 \mathrm{~min}$ at $0^{\circ} \mathrm{C}$, the pellet was washed with distilled water and recentrifuged. The pellet was then dried to constant weight at $90^{\circ} \mathrm{C}$ in pre-weighed vials.

Extraction of phenazines. Phenazines were extracted from acidified cultures $(\mathrm{pH} 2) 24 \mathrm{~h}$ after growth had ceased by adding an equal volume of chloroform and shaking on a gyratory shaker for at least $6 \mathrm{~h}$. The chloroform layer 
was then separated from the aqueous layer using Whatman phase-separating paper and the extraction repeated.

Estimation of phenazines. Because of the difficulty in purifying enough of each phenazine to measure its molar absorbance coefficients it was only possible to estimate total phenazine production. Therefore phenazines were estimated spectrophotometrically in chloroform solution. Phenazine 1,6-dicarboxylate and phenazine 1carboxylate were estimated by measuring absorbance at $254 \mathrm{~nm}$ and $250 \mathrm{~nm}$, respectively. The absorption maximum of 1,6-dihydroxyphenazine occurred at $273 \mathrm{~nm}$ and that of iodinin at $290 \mathrm{~nm}$. 1,6-Dihydroxyphenazine $N$-oxide was estimated by measuring its absorbance at $283 \mathrm{~nm}$.

By separating phenazines on silica TLC plates run in chloroform/acetic acid (9:1, v/v) (Byng \& Turner, 1975) and scanning the plates at the above wavelengths it was shown that each of these wavelengths was characteristic of one phenazine fraction, and that there was no interference by other products absorbing at these wavelengths.

\section{RESULTS}

\section{Effect of medium composition on phenazine production}

Over a range of carbon ( 0 to $15 \mathrm{~g}$ threonine $\left.\mathrm{l}^{-1}\right)$, sulphate (0 to $\left.10 \mathrm{mg} \mathrm{MgSO}_{4} \mathrm{l}^{-1}\right)$, magnesium ( 0 to $1 \mathrm{mg} \mathrm{MgSO} \mathrm{M}^{-1}$ ), potassium ( 0 to $25 \mathrm{mg} \mathrm{KH}_{2} \mathrm{PO}_{4} \mathrm{l}^{-1}$ ) and phosphate (0 to $5 \mathrm{mg}$ $\mathrm{KH}_{2} \mathrm{PO}_{4} 1^{-1}$ ) concentrations, phenazine production in wild-type $P$. phenazinium paralleled growth, i.e. a depletion of none of the components tested caused increased production. Under conditions of nitrogen and sulphate depletion, however, phenazine 1-carboxylate (normally present in only trace amounts) was the major phenazine formed rather than iodinin.

When carbon-depleted medium containing $0.5 \mathrm{~g}$ threonine $1^{-1}$ was supplemented with glycerol $\left(0\right.$ to $\left.10 \mathrm{~g} \mathrm{l}^{-1}\right)$, to produce nitrogen-depleted conditions, maximal phenazine production

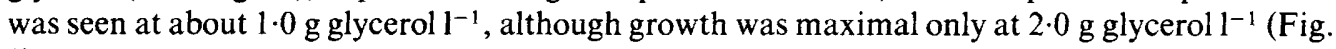
2).

The addition of $\left(\mathrm{NH}_{4}\right)_{2} \mathrm{SO}_{4}\left(1\right.$ to $\left.10 \mathrm{~g} \mathrm{l}^{-1}\right)$ to L-threonine medium ( $\mathrm{pH} \mathrm{5.5)} \mathrm{dramatically}$ reduced iodinin production without affecting growth (Fig. 3). Over this range of $\left(\mathrm{NH}_{4}\right)_{2} \mathrm{SO}_{4}$ concentrations other phenazines were produced in only trace amounts.

\section{Phenazine production in continuous culture}

During carbon-limited growth of mutant D34, phenazine production occurred over a wide range of dilution rates, peaking at $0 \cdot 11 \mathrm{~h}^{-1}$. Cell mass remained constant over a similar range of dilution rates (Fig. 4a). Although cell mass was unaffected at higher rates, phenazine production dropped to virtually zero at a growth rate of $0.25 \mathrm{~h}^{-1}$. Under nitrogen- and magnesium-limited conditions the decrease in phenazine production at high dilution rates corresponded to a decline in cell mass (Fig. $4 b, c$ ). Under all these limiting conditions the decline of phenazine production at low dilution rates corresponded to an increase in cell mass. This was especially pronounced under magnesium-limiting conditions.

Phenazine production was also maintained over a wide range of oxygen tensions on carbonlimiting medium (Fig. 5). Although it declined at saturation levels below $10 \%$ some phenazine production still occurred at the lowest oxygen saturation tested $(0.4 \%)$. However, cell mass remained steady over this range of oxygen saturation.

In batch culture the addition of phenylalanine $\left(0 \cdot 1\right.$ to $\left.0 \cdot 5 \mathrm{~g} \mathrm{l}^{-1}\right)$ to carbon-depleted medium appears to inhibit both phenazine production and growth (G.S. Byng, University of Liverpool, U.K., unpublished results). To test the possibility that decreased phenazine production was not an indirect effect of growth inhibition the phenomenon was studied in continuous culture. In carbon-limiting medium containing a low concentration of phenylalanine $\left(0 \cdot 1 \mathrm{~g}^{-1}\right)$, phenazine production was confined to a narrower range of growth rates than in unsupplemented medium (compare Fig. $4 a$ and $d$ ). Cell mass was comparable with that on medium lacking phenylalanine.

\section{DISCUSSION}

Nitrogen, supplied as $\left(\mathrm{NH}_{4}\right)_{2} \mathrm{SO}_{4}$, had an inhibitory effect on phenazine production in $P$. phenazinium. Nitrogen catabolite repression has long been known to regulate secondary metabolism is certain cases. Trihydroxytoluene production in Aspergillus fumigatus only occurs 


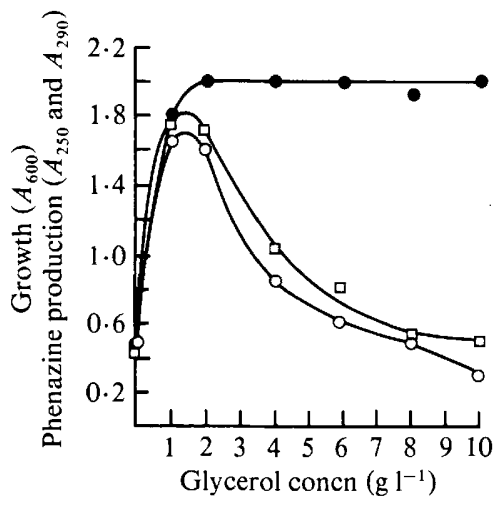

Fig. 2. Effect of supplementary glycerol in carbon-depleted medium on phenazine production. Cultures of $P$. phenazinium wild-type were grown in carbon-depleted medium containing $0.5 \mathrm{~g}$ threonine $1^{-1}$ and mineral salts and various concentrations of glycerol were added so that medium became nitrogen-depleted. Maximal growth $\left(A_{600}, O\right)$ and phenazine production $\left(A_{250}, \square ; A_{290}, O\right)$ of these cultures were measured as described in Methods.

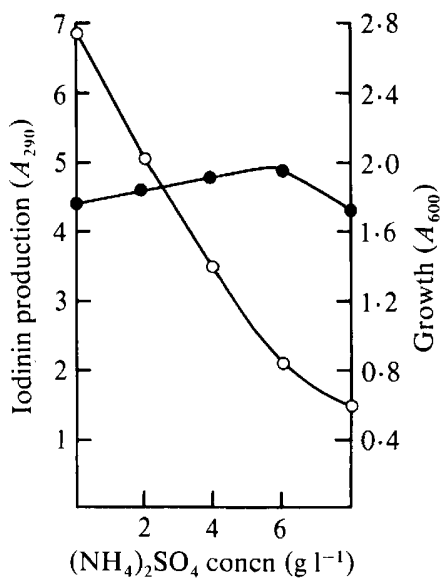

Fig. 3. Effect of supplementary $\left(\mathrm{NH}_{4}\right)_{2} \mathrm{SO}_{4}$ on iodinin production by $P$. phenazinium. Cultures of $P$. phenazinium wild-type were grown on threonine/mineral salts carbon-depleted medium with various amounts of $\left(\mathrm{NH}_{4}\right)_{2} \mathrm{SO}_{4}$ added. Maximal growth $\left(A_{600}, \mathrm{O}\right)$ and iodinin production $\left(A_{290}, O\right)$ were measured as described in Methods.

when nitrogen in the medium is exhausted, and the addition of ammonium salts completely inhibits production without affecting growth or $\mathrm{pH}$ (Ward \& Packter, 1974). The peak of phenazine production seen in glycerol-supplemented medium as the culture becomes nitrogendepleted also suggests that the balance of carbon and nitrogen is important in regulating phenazine production.

Regulation of phenazine production in $P$. phenazinium differed from that in $P$. aeruginosa in that no inhibition by phosphate occurred. The narrowing of the range of growth rates supporting good phenazine production by phenylalanine in $P$. phenazinium also differs from $P$. aeruginosa, where tyrosine and phenylalanine enhance both phenazine production and growth (Labeyrie \& Neuzil, 1981).

Phenazine production in continuous culture over a wide range of growth rates suggested that secondary metabolism is not restricted to the physiologically similar late growth phase and stationary phase in all cases. Changes in medium composition altered the optimum growth rate for phenazine production, as well as the pattern of phenazines produced. Such evidence supports 

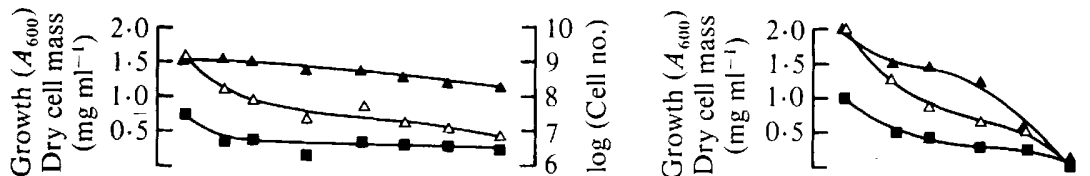

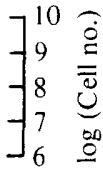
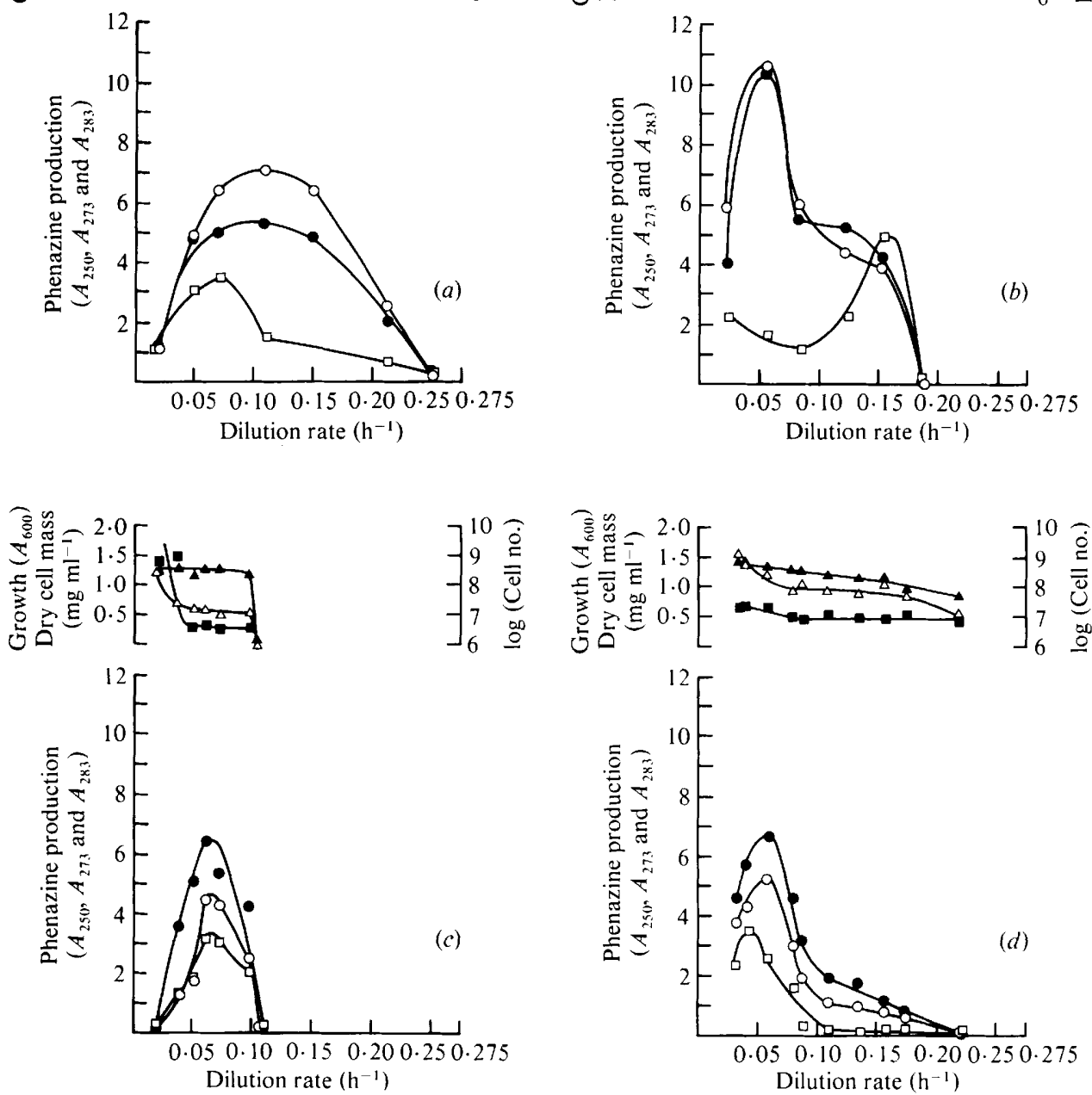

Fig. 4. Continuous culture of $P$. phenazinium mutant D34. Phenazine production $\left(A_{250}, O ; A_{273}, \bigcirc\right.$ : $\left.A_{283}, \square\right)$, growth $\left(A_{600}, \triangle\right)$, dry cell mass $(\boldsymbol{\square})$ and log cell number $(\boldsymbol{\Lambda})$ were measured under various growth conditions: $(a)$ carbon-limiting, $(b)$ nitrogen-limiting, $(c)$ magnesium-limiting and $(d)$ with the addition of phenylalanine $\left(0 \cdot 1 \mathrm{~g} \mathrm{l}^{-1}\right)$ to carbon-limiting medium.

the opinion of Demain et al. (1978) that secondary metabolites cannot be defined as compounds produced when growth has ceased but that the timing of secondary metabolite formation is a function of nutritional requirements. From the results it seems that when resources are limited, i.e. at low growth rates, the advantage of phenazine production is outweighed by the need to conserve cell resources and so cell mass is maintained at the expense of phenazine production. The increase in cell mass at these growth rates may be due to a shift in metabolism from phenazine production to the synthesis of intracellular metabolites, probably storage compounds. At the other extreme, the decline in phenazine production at high growth rates may reflect the fact that phenazines are unnecessary to the cell when resources are plentiful and little competition with other micro-organisms exists. At this stage this remains speculative since the function of phenazines in the producing cell remains unknown. 


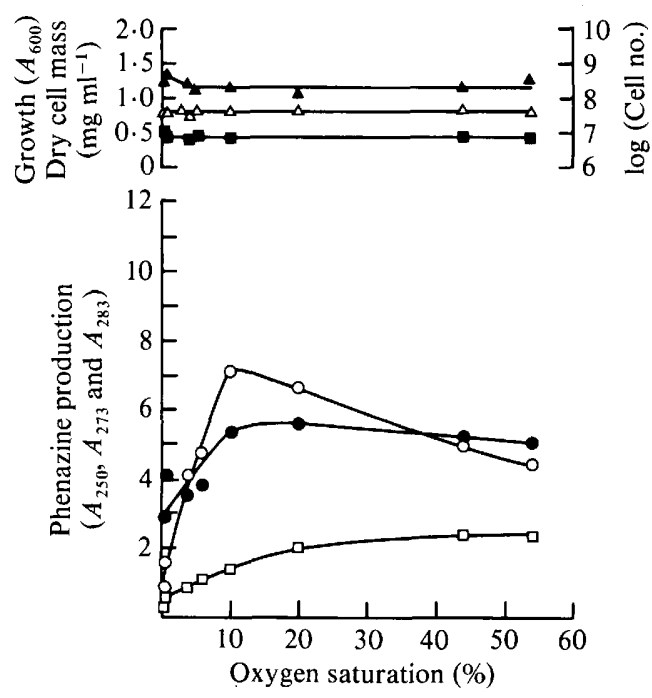

Fig. 5. Continuous culture of $P$. phenazinium mutant D34 under different oxygen tensions. Phenazine production $\left(A_{250}, \bigcirc ; A_{273}, \bigcirc ; A_{283}, \square\right)$, growth $\left(A_{600}, \triangle\right)$, dry cell mass $(\square)$ and log cell number $(\mathbf{\Lambda})$ were measured at steady states at a dilution rate of $0 \cdot 11 \mathrm{~h}^{-1}$ on carbon-limiting medium.

The work of A. J.M. M. was supported by an MRC Research Studentship. The interest and support of Professor T. W. Goodwin F.R.S. is gratefully acknowledged.

\section{REFERENCES}

Bell, S. C. \& Turner, J. M. (1973). Iodinin biosynthesis by a pseudomonad. Biochemical Society Transactions 1, 751-753.

Byng, G. S. \& Turner, J. M. (1975). Phenazine biosynthesis by a pseudomonad. Biochemical Society Transactions 3, 742-744.

BYNG, G. S. \& TURNER, J. M. (1976). Isolation of pigmentation mutants of Pseudomonas phenazinium. Journal of General Microbiology 97, 57-62.

BYNG, G. S. \& TURNER, J. M. (1977). Incorporation of $\left[{ }^{4} \mathrm{C}\right]$ shikimate into phenazines and their further metabolism by Pseudomonas phenazinium. Biochemical Journal 164, 139-145.

Calhoun, D. H., Carson, M. \& Jensen, R. A. (1972). The branch point metabolite for pyocyanine biosynthesis in Pseudomonas aeruginosa. Journal of General Microbiology 72, 581-583.

Demain, A. L., Kennel, Y. M. \& Aharonowitz, Y. (1978). Carbon catabolite regulation of secondary metabolism. Symposia of the Society for General Microbiology 29, 163-185.

HadiJpetrou, L. P., Gerrits, J. P., Teulings, F. A. G. \& Stouthamer, A. H. (1964). Relation between energy production and growth of Aerobacter aerogenes. Journal of General Microbiology 36, 139150.

INGLedew, W. M. \& CAMpbell, J. J. R. (1969). A new resuspension medium for pyocyanine production. Canadian Journal of Microbiology 15, 595-598.

Kanner, D., Gerber, N. M. \& Bartha, R. (1978). Pattern of phenazine pigment production by a strain of Pseudomonas aeruginosa. Journal of Bacteriology 134, 690-692.

KORTH, H. (1973). Carbon source regulation of the phenazine- $\alpha$-carboxylic acid synthesis in Pseudomonas aureofaciens. Archiv für Mikrobiologie 92, 175177.

KORTH, H. (1974). Mixed carbon source effect in the phenazine- $\alpha$-carboxylic acid synthesis and the aromatic pathway in Pseudomonas spp. Archiv für Mikrobiologie 97, 245-252.

LABEYRIE, S. \& NeUZIL, E. (1981). Influence of supplementary tyrosine or phenylalanine on bacterial growth and pigmentation of Pseudomonas aeruginosa. Annales de Microbiologie 132A, 31-40.

Longley, R. P., Halliwell, J. E., Campbell, J. J. R. \& INGLEDEW, W. M. (1972). The branchpoint of pyocyanine biosynthesis. Canadian Journal of Microbiology 18, 1357-1368.

WARD, A. C. \& PACKTER, N. M. (1974). Relationship between fatty acid and phenol synthesis in Aspergillus fumigatus. European Journal of Biochemistry 46, 323-333. 